\title{
HCl ppb-level detection based on QEPAS sensor using a low resonance frequency quartz tuning fork
}

\author{
Yufei Ma ${ }^{1,2, *}$, Ying $\mathrm{He}^{1}$, Xin $\mathrm{Yu}^{1}$, Deying $\mathrm{Chen}^{1}$, Rui Sun ${ }^{3}$, Frank K. Tittel ${ }^{2}$ \\ ${ }^{1}$ National Key Laboratory of Science and Technology on Tunable Laser, Harbin Institute of \\ Technology, Harbin 150001, China \\ ${ }^{2}$ Department of Electrical and Computer Engineering, Rice University, 6100 Main Street, \\ Houston, Texas 77005, USA \\ ${ }^{3}$ Post-doctoral Mobile Station of Power Engineering and Engineering Thermophysics, \\ Harbin Institute of Technology, Harbin 150001, China \\ "e-mail: mayufei926@163.com
}

\begin{abstract}
In this report, a sensitive quartz-enhanced photoacoustic spectroscopy (QEPAS) based hydrogen chloride $(\mathrm{HCl})$ sensor using a quartz tuning fork (QTF) with a resonance frequency of $30.72 \mathrm{kHz}$ was demonstrated for the first time. A fiber-coupled, continuous wave (CW), distributed feedback (DFB) diode laser emitting at $1.74 \mu \mathrm{m}$ was employed as the excitation laser source. Wavelength modulation spectroscopy and a 2nd harmonic detection technique were used to reduce the sensor background noise. An acoustic micro-resonator (mR) was added to the QTF sensor architecture to improve the signal amplitude. For the reported $\mathrm{HCl}$ sensor system operating at atmospheric pressure, a $550 \mathrm{ppbv}$ (parts per billion by volume) minimum detection limit at $5739.27 \mathrm{~cm}^{-1}$ was achieved when the modulation depth and the data acquisition time were set to $0.23 \mathrm{~cm}^{-1}$ and $1 \mathrm{sec}$, respectively. The ppb-level detection sensitivity and robust design of the QEPAS technique makes it suitable for use in environmental monitoring and other applications.
\end{abstract}

Key words: Trace gas detection; QEPAS; Hydrogen chloride ( $\mathrm{HCl})$; Quartz tuning fork

\section{Introduction}

Hydrogen chloride $(\mathrm{HCl})$, a toxic gas, is primarily produced by burning fuels that contain chlorine and incinerating waste that contains plastics [1,2]. $\mathrm{HCl}$ is not only a source of dioxin and acid rain, but also has been identified as hazardous in industrial workplaces, with a short period exposure limit of $5 \mathrm{ppm}$. Furthermore, $\mathrm{HCl}$ has performed an important role in photochemistry [3] and plasma etching [4]. Hence, there is a significant need for sensitive detection of $\mathrm{HCl}$ gas concentrations at sub-ppm levels.

Laser absorption spectroscopy (LAS) has the advantages that include fast response, non-invasive, highly sensitive and selective detectio compared with 
chemical sensors which are currently widely used for $\mathrm{HCl}$ detection [5]. Tunable diode laser absorption spectroscopy (TDLAS) was adopted for $\mathrm{HCl}$ measurements [4,6] and a minimum detection limit (MDL) of $45 \mathrm{ppm}$ was obtained [6]. In order to further improve the detection sensitivity, a multi-pass gas cell (MPGC) is usually used in the TDLAS technique, where the effective optical path length is typically tens and even hundreds of meters in order to reach a detection limit of the analyte trace gas species at ppb concentration levels [7-9]. However for ultra-high sensitive measurements these sensors are usually bulky and costly due to the large size of a MPGC and the increased number of optical components that are needed for laser beam alignment and detection. Photoacoustic spectroscopy (PAS) is another effective trace gas sensor technology which employs a broadband microphone for acoustic wave detection. When the output of a near-infrared semiconductor laser is absorbed by a gas sample, the absorbed energy is transformed to heat energy by non-radiative processes, and will subsequently result in an increase of the local temperature and pressure in the sample. Therefore the absorption of a modulated near-infrared laser beam in a gas sample leads to the generation of an acoustic wave. The intensity of the acoustic wave is related to the sample concentration which can be detected by a sensitive microphone. However, most microphone-based PAS cells have a low resonance frequency $(<2 \mathrm{kHz})$, which makes such cells more sensitive to environmental and sample gas flow noise [10].

A modification of the conventional PAS is the quartz-enhanced photoacoustic spectroscopy (QEPAS) technique which was first reported in 2002 [11]. This technique uses a commercially available millimeter sized piezoelectric quartz tuning fork (QTF) as an acoustic wave transducer. The high Q-factor and narrow resonance frequency band of QTF improve the QEPAS selectivity and immunity to environmental acoustic noise. The primary noise source of QEPAS is limited by the fundamental Johnson thermal noise of the QTF. The QEPAS signal amplitude $S$ is given by equation (1) [12].

$$
S \propto \frac{\alpha P Q}{f_{0}}
$$

where $\alpha$ is the absorption coefficient, $P$ is the optical power, $Q$ is the Q-factor of QTF, $f_{0}$ is the resonance frequency of QTF. From Equ. (1) it is seen that the QEPAS signal amplitude $S$ is inversely proportional to the QTF resonance frequency. This is due to the fact that a QTF with a smaller $f_{0}$ will result in a longer effective integration time, which increases the QEPAS signal. In QEPAS, commercially available QTFs with a $f_{0}$ of $\sim 32.76 \mathrm{kHz}$ are usually employed, and only since 2013 the use of custom QTFs in QEPAS based sensor systems were reported [13-15].

In this paper, a high sensitive $\mathrm{HCl}$ trace gas sensor based on QEPAS technology using a QTF with $f_{0}$ of $30.72 \mathrm{kHz}$ was demonstrated for the first time. A pigtailed, near infrared, continuous wave (CW), distributed feedback (DFB) diode laser emitting at $1.74 \mu \mathrm{m}$ was employed as the excitation source. This sensor resulted in a $550 \mathrm{ppbv}$ MDL for a $1 \mathrm{sec}$ data acquisition time. The ppb-level sensitivity and robust design of 
QEPAS method makes it suitable for use in environmental monitoring and other applications.

\section{Experimental Setup}

\subsection{DFB diode laser characterization}

The simulation of the $\mathrm{HCl}$ absorption line strength based on the HITRAN 08 database is shown in Fig. 1. From Fig. 1(a), we can determine that the strongest $\mathrm{HCl}$ absorption region of fundamental rotational-vibrational band is located in the $3.5 \mu \mathrm{m}$ ( $1 v$-band), which can be accessed using interband cascade lasers (ICLs) in the 3 4 $\mu \mathrm{m}$ wavelength region [16]. However, diode lasers with emission wavelength of $<3 \mu \mathrm{m}$ have several advantages, such as compactness, significant lower cost and higher optical power. Therefore, in this research, a $1.74 \mu \mathrm{m}$ CW-DFB fiber-coupled diode laser (NEL Corp., Japan) in a 14-pin butterfly package that included a thermoelectric control (TEC) was employed as the excitation source to access the $\mathrm{HCl} 2 v$ overtone band located at $1.7 \mu \mathrm{m}$. Using the HITRAN data base we identified the $1742.38 \mathrm{~nm}$ (5739.27 $\mathrm{cm}^{-1}$ ) absorption line as the strongest in the $1.7 \mu \mathrm{m}$ spectral range (see Fig. 1 (b)) at a temperature of $296 \mathrm{~K}$, standard atmospheric pressure and an optical path length of $1 \mathrm{~cm}$ for $5000 \mathrm{ppm} \mathrm{HCl}$. This absorption line is free from spectral interference of other molecules. The absorption coefficient is $\sim 0.003 \mathrm{~cm}^{-1}$ which is sufficient to provide a strong QEPAS signal.

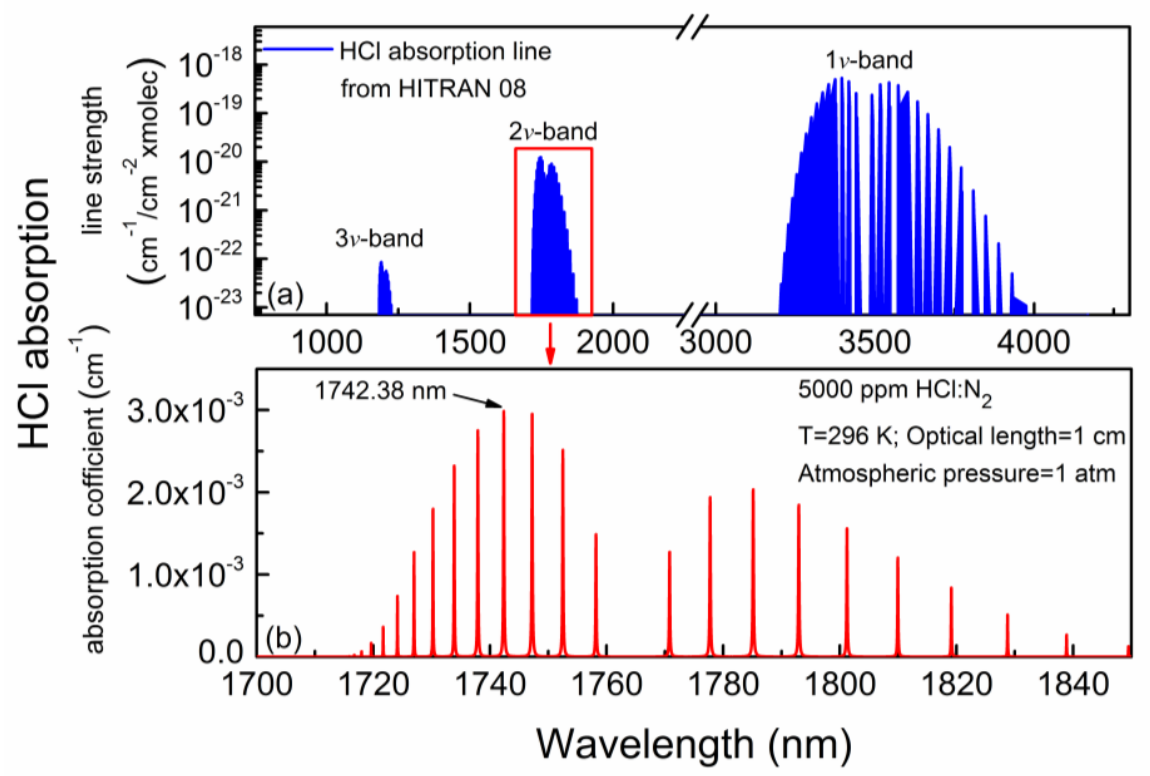

Fig. 1 HITRAN based simulation of $\mathrm{HCl}$ absorption spectra: (a) absorption line strength; (b) absorption coefficient at $296 \mathrm{~K}$, standard atmospheric pressure, and an optical path length of $1 \mathrm{~cm}$ for 5000 ppm HCl: $\mathrm{N}_{2}$

The diode laser output performance was verified and is shown in Fig. 2. The experimentally determined temperature and current tuning coefficients of the laser were $-0.43 \mathrm{~cm}^{-1} /{ }^{\circ} \mathrm{C}$ and $-0.039 \mathrm{~cm}^{-1} / \mathrm{mA}$, respectively. The maximum optical power emitted by the diode laser operating at temperature of $21{ }^{\circ} \mathrm{C}$ and an injection current of $120 \mathrm{~mA}$ was $\sim 16.24 \mathrm{~mW}$ (see Fig. 2(b)). The DFB diode laser can be current tuned 
to target the $\mathrm{HCl}$ absorption line of the first overtone at $1742.38 \mathrm{~nm}\left(5739.27 \mathrm{~cm}^{-1}\right)$, which is free from spectral interference of other molecules.
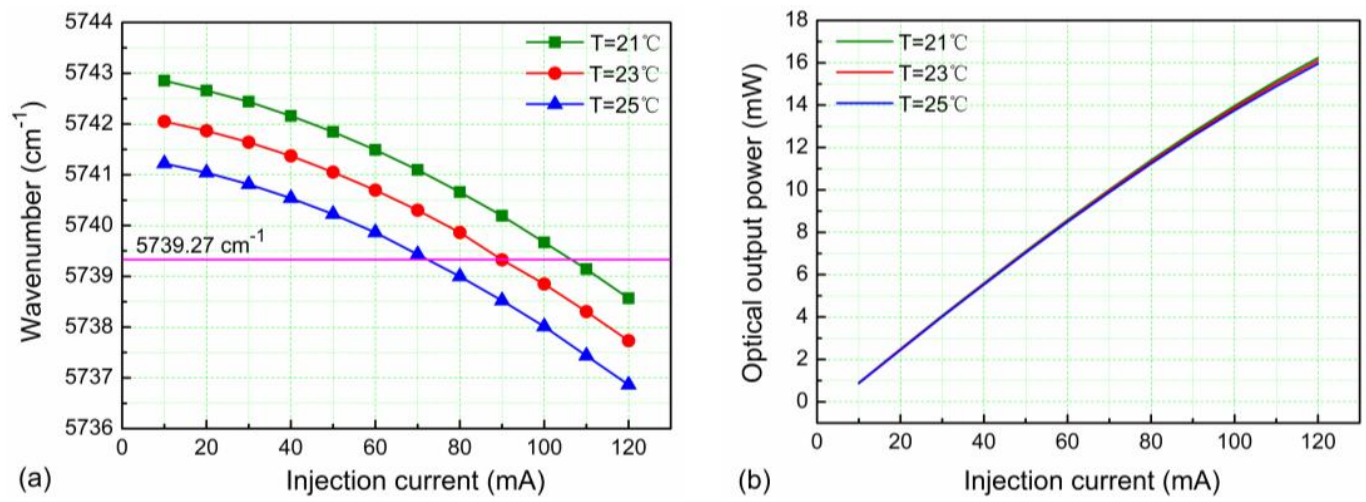

Fig. 2 1.74 $\mu \mathrm{m}$ CW-DFB fiber-coupled diode laser output performance: (a) current tuning at different operating temperatures; (b) optical output power as a function of injection current

\subsection{Sensor configuration}

A schematic of the QEPAS based sensor platform is shown in Fig. 3. The laser beam was collimated by using a fiber collimator (FC) and subsequently focused between the QTF prongs inside acoustic detection module (ADM) by means of a plano-convex $\mathrm{CaF}_{2}$ lens (L) with a $50 \mathrm{~mm}$ focal length. After passing through the ADM the laser beam was directed to an optical power meter (PS19Q, Coherent) and used for alignment verification of the QEPAS based sensor system. A QTF with $f_{0}$ of $30.72 \mathrm{kHz}$ was employed as the acoustic transducer. The geometry of QTF is listed in Table 1. For comparison, a QTF with $f_{0}$ of $32.768 \mathrm{kHz}$ is also listed in Table 1. Wavelength modulation spectroscopy (WMS) with 2nd harmonic detection [17] was utilized for sensitive concentration measurements. Modulation of the laser current was performed by applying a sinusoidal dither to the direct current ramp of the diode laser at half of the QTF resonance frequency $\left(f=f_{0} / 2 \approx 15.36 \mathrm{kHz}\right)$. The piezoelectric signal generated by the QTF was detected by a low noise transimpedance amplifier (TA) with a $10 \mathrm{M} \Omega$ feedback resistor and converted into a voltage, which was transferred to a custom built control electronics unit (CEU). The CEU provides the following three functions: 1) measurement of the QTF parameters, i.e. the quality factor $Q$, dynamic resistance $R$, and resonant frequency $f_{0} ; 2$ ) modulation of the laser current at the frequency $f=f_{0} / 2$; and 3 ) measurement of the $2 f$ component generated by the QTF. The HCl-QEPAS sensor performance was evaluated at different $\mathrm{HCl}$ concentrations. Two mass flow controllers with a mass flow uncertainty of $3 \%$ were used to dilute 5000 ppmv (parts in $10^{6}$ by volume) $\mathrm{HCl}$ in nitrogen $\left(\mathrm{N}_{2}\right)$. The measurements were carried out at atmospheric pressure and room temperature $\left(\sim 20^{\circ} \mathrm{C}\right)$.

Table 1. Parameters of geometries for QTFs.

\begin{tabular}{ccccc}
\hline QTF with $f_{0}(\mathrm{kHz})$ & Length $(\mathrm{mm})$ & Width $(\mathrm{mm})$ & Thickness $(\mathrm{mm})$ & Gap $(\mathrm{mm})$ \\
\hline 30.72 & 3.9 & 0.62 & 0.36 & 0.32 \\
32.768 & 3.6 & 0.6 & 0.36 & 0.3 \\
\hline
\end{tabular}




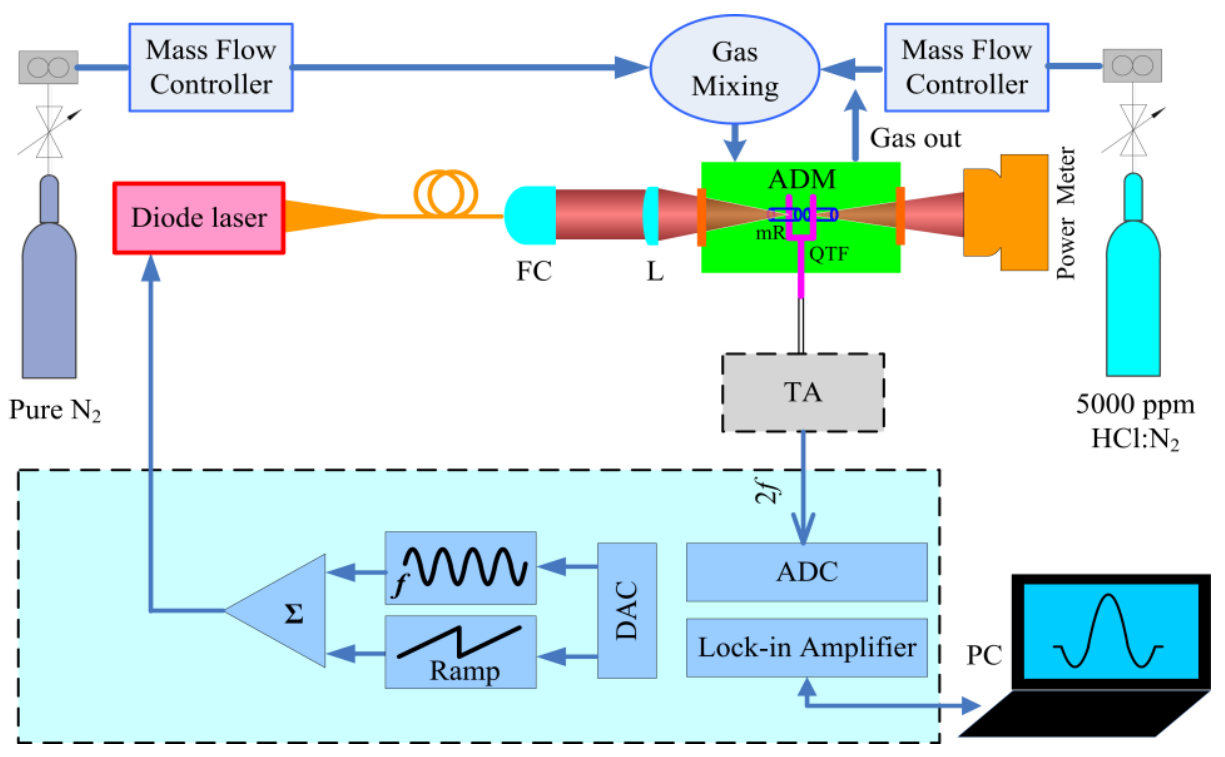

Fig. 3 Schematic configuration of a QEPAS system for $\mathrm{HCl}$ detection

\section{Experimental Results and Discussion}

The HCl-QEPAS sensor performance using a basic QTF was evaluated first. A certified mixture of 5000 ppm $\mathrm{HCl}: \mathrm{N}_{2}$ was used. The impact of distance (L) between the laser beam and the top of QTF prongs on QEPAS signal level was investigated and the experimental results are shown in Fig. 4. An insert in Fig. 4 displays the laser beam, the QTF and the parameter L. The modulation depth of laser wavelength was set to $0.16 \mathrm{~cm}^{-1}$. The $2 f \mathrm{HCl}$-QEPAS signal amplitude increased rapidly with $\mathrm{L}$ when it was $<0.6 \mathrm{~mm}$. The peak region of the $2 f$ signal amplitude appeared in the range of $\mathrm{L}$ from $0.6 \mathrm{~mm}$ to $1.3 \mathrm{~mm}$. With a further increase of $\mathrm{L}$, the signal amplitude decreased due to the more challenging QTF prong vibration when the acoustic wave source is at the bottom of the QTF prongs. In the following experiments, an optimum value of $\mathrm{L}$ of $1 \mathrm{~mm}$ was chosen to achieve the maximum QEPAS signal amplitude.

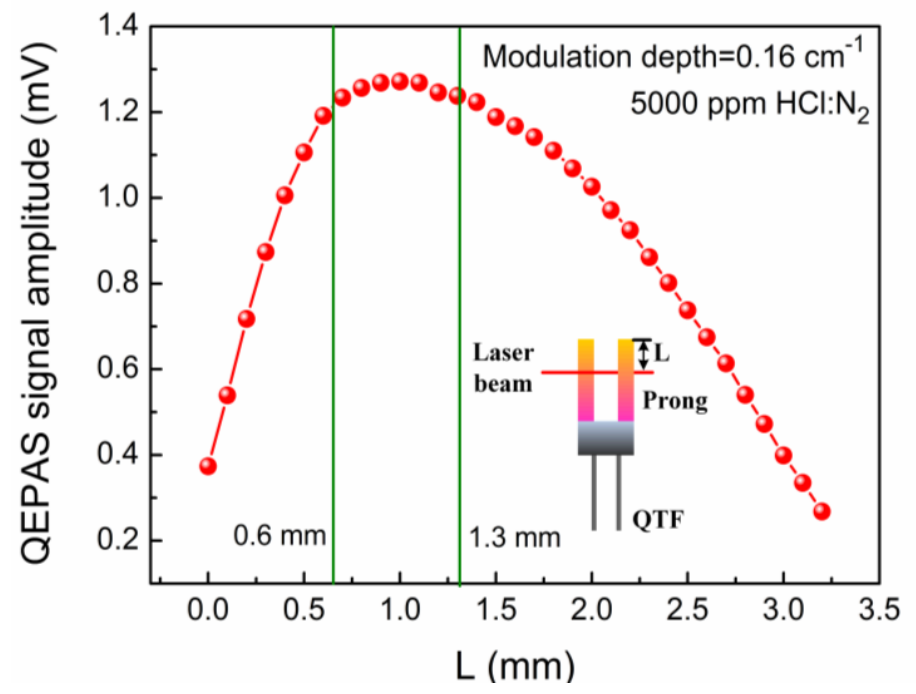

Fig. $4 \mathrm{HCl}$-QEPAS signal amplitude as a function of $\mathrm{L}$ at a modulation depth of $0.16 \mathrm{~cm}^{-1}$ 


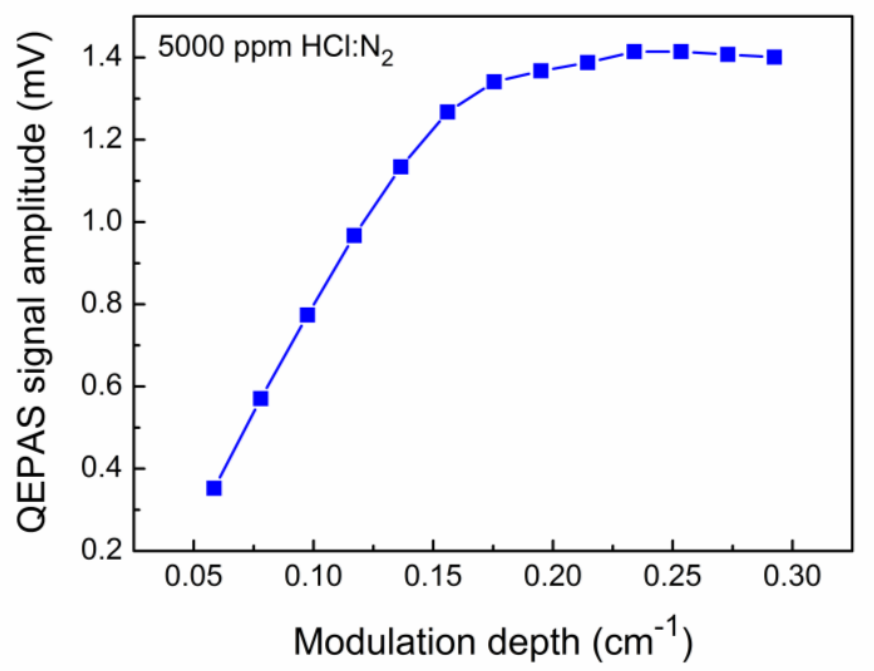

Fig. $5 \mathrm{HCl}$-QEPAS signal amplitude as a function of modulation depth for a $5000 \mathrm{ppm} \mathrm{HCl}: \mathrm{N}_{2}$ mixture

The laser wavelength modulation depth was optimized in order to improve the $2 f$ QEPAS signal amplitude. The dependence of the QEPAS signal amplitude as a function of the laser wavelength modulation depth is depicted in Fig. 5. The QEPAS signal amplitude increased with the modulation depth, but when the modulation depth was higher than $0.23 \mathrm{~cm}^{-1}$, no further significant change was observed. Therefore, a modulation depth of $0.23 \mathrm{~cm}^{-1}$ was found to be optimum.

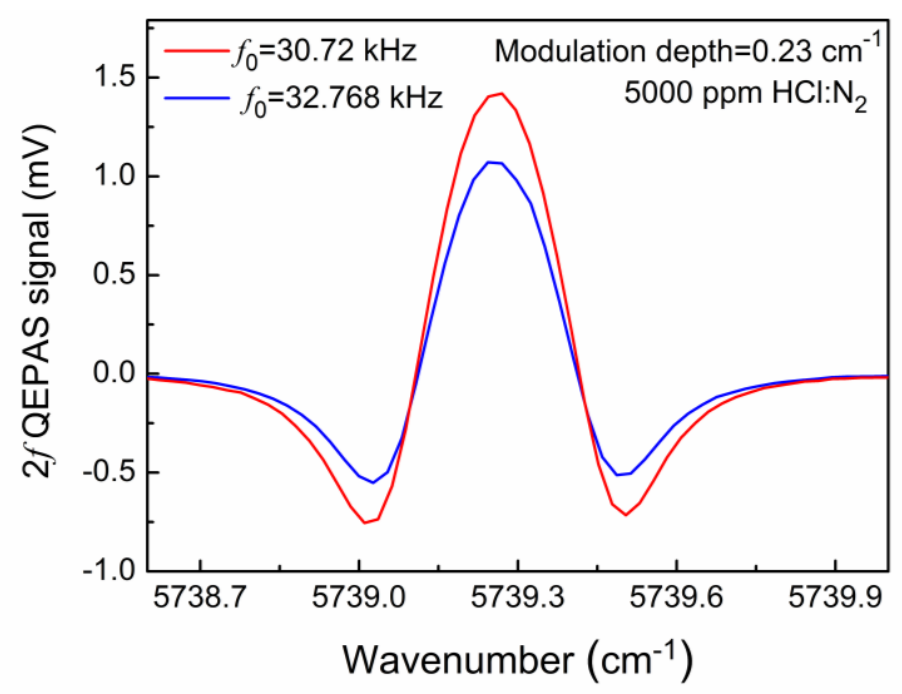

Fig. $62 f \mathrm{HCl}$-QEPAS signal for two QTFs with different $f_{0}$ of $30.72 \mathrm{kHz}$ and $32.768 \mathrm{kHz}$ for the same experimental conditions

In our investigations, a QTF with a $f_{0}$ of $30.72 \mathrm{kHz}$ was used. According to Equ. (1), a QTF with a low $f_{0}$ is advantageous to obtain a large QEPAS signal. Therefore, a comparison was made to confirm the advantages of using a QTF with $f_{0}$ of $30.72 \mathrm{kHz}$ when compared to a standard QTF with a $f_{0}$ of $32.768 \mathrm{kHz}$. The experiments were carried out under the same conditions and the results are displayed in Fig. 6. We found that, compared to a QTF with $f_{0}$ of $32.768 \mathrm{kHz}$, the QEPAS signal increased 1.3 times 
when a QTF with $f_{0}$ of $30.72 \mathrm{kHz}$ was used. Two aspects contributed to this QEPAS signal increase. One is a low $f_{0}$ of $30.72 \mathrm{kHz}$. The other is an increased Q factor. The Q factor for $32.768 \mathrm{kHz}$ QTF was 7453 and that for $30.72 \mathrm{kHz}$ QTF was 8996. The Q factor increased by $20 \%$ and according to Equ. (1) contributed to the QEPAS signal enhancement.

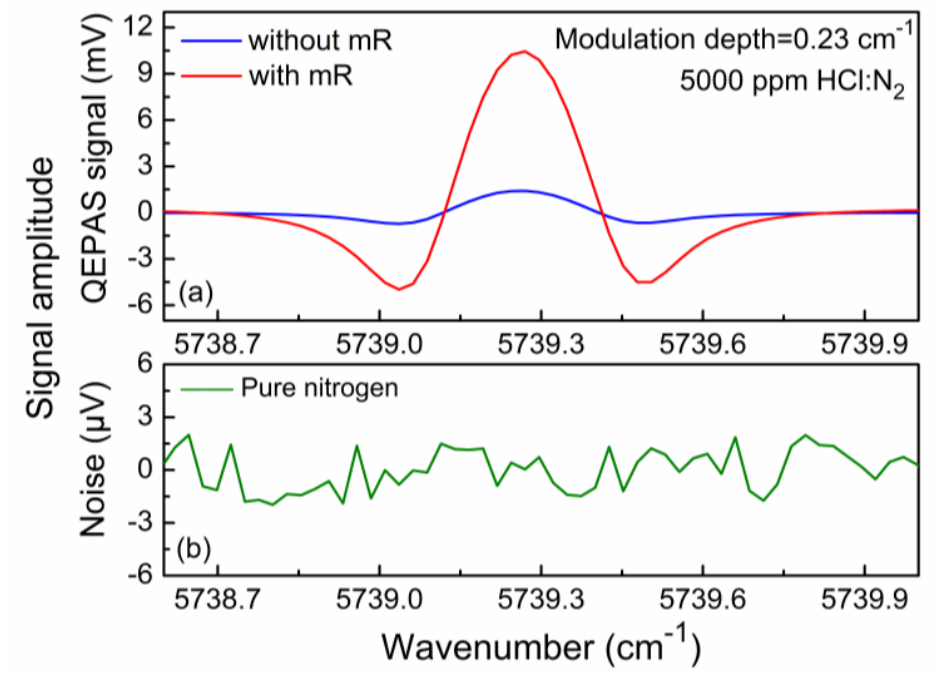

Fig. 7 Signal amplitude: (a) $2 f \mathrm{HCl}$-QEPAS signal without and with $\mathrm{mR}$ obtained with a modulation depth of $0.23 \mathrm{~cm}^{-1}$ and using a QTF with $f_{0}$ of $30.72 \mathrm{kHz}$;

(b) pure $\mathrm{N}_{2}$ for noise determination

A significant enhancement of the QEPAS signal can be achieved when two metallic tubes acting as a micro-resonator $(\mathrm{mR})$ are added to the QTF sensor architecture $[18,19]$. The optimum length $L$ of metallic tubes should be in the range of $\lambda_{s} / 4<L<\lambda_{s} / 2$, where $\lambda_{s}$ is the sound wavelength. For a QTF with $f_{0}$ of $30.72 \mathrm{kHz}$, the optimum length should be $2.8 \mathrm{~mm}<L<5.5 \mathrm{~mm}$. In this experiment, the length and inner diameter of stainless tubes were selected to be $4 \mathrm{~mm}$ and $0.5 \mathrm{~mm}$ respectively in order to compose the $\mathrm{mR}$. The gaps between the QTF and $\mathrm{mR}$ tubes were chosen to be $25 \mu \mathrm{m}$. The measured $2 f$ QEPAS signals with and without $\mathrm{mR}$ at a modulation depth of $0.23 \mathrm{~cm}^{-1}$ is shown in Fig. 7(a). The QEPAS signal was enhance 7.5-fold as a result of the addition of the two mR tubes. Fig. 7(b) depicts the background signal measured when the ADM was flushed with ultra high purity $\mathrm{N}_{2}$. The background signal was $1.15 \mu \mathrm{V}$ and is primarily determined by the fundamental thermal noise of the QTF. The QTF thermal noise can be expressed in terms of its root mean square (rms) voltage noise [12], and is shown in equation (2), where $k_{B}$ is the Boltzmann constant, $T$ is QTF temperature, $R_{g}$ is the value of the feedback resistor used in a transimpedance amplifier located close to the ADM, $R$ is the equivalent electrical parameter of resistance for the QTF when it is represented by an equivalent series resonant circuit. The measured equivalent resistance for the QTF was $643 \mathrm{k} \Omega$ and the calculated thermal noise was $1.41 \mu \mathrm{V}$. Based on the data depicted in Fig. 7 the $1 \sigma$ MDL of the HCl-QEPAS sensor is $550 \mathrm{ppbv}$ for a $1 \mathrm{sec}$ data acquisition time. The calculated normalized noise equivalent absorption coefficient (NNEA) is $1.76 \times 10^{-8}$ $\mathrm{cm}^{-1} \mathrm{~W} / \sqrt{ } \mathrm{Hz}$. 


$$
\sqrt{V_{r m s}^{2}}=R_{g} \sqrt{\frac{4 k_{B} T}{R}} \sqrt{\Delta f}
$$
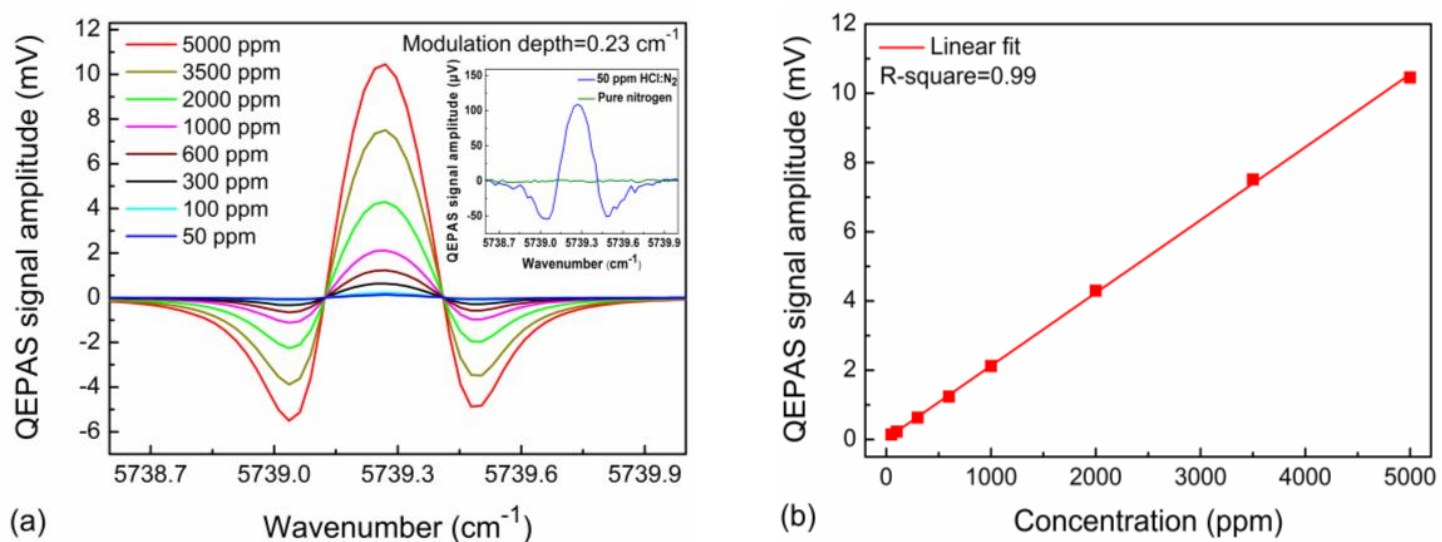

Fig. 8(a): QEPAS signal amplitude at different $\mathrm{HCl}$ concentrations and a modulation depth of 0.23

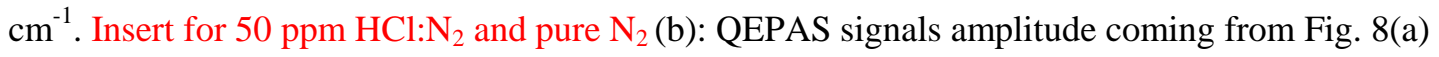
as a function of $\mathrm{HCl}$ concentrations

To verify the linear response of the QEPAS based $\mathrm{HCl}$ sensor platform a calibration mixture of $5000 \mathrm{ppm} \mathrm{HCl}: \mathrm{N}_{2}$ was diluted with dry $\mathrm{N}_{2}$ down to $50 \mathrm{ppm}$ $\mathrm{HCl}$ concentration levels (see Fig. 8(a)). The data acquisition time for these measurements was set to $1 \mathrm{sec}$. The measured QEPAS signal amplitude as a function of $\mathrm{HCl}$ concentrations are plotted in the Fig. 8(b). The calculated R-square value, which represents how well the regression line approximates real data points, is equal to $\sim 0.99$ after a linear fitting procedure. This implies that the sensor system exhibits an excellent linearity response of the monitored $\mathrm{HCl}$ concentration levels. Based on the data depicted in the insert of Fig. 8(a), a $1 \sigma$ MDL of 526 ppbv for the HCl-QEPAS sensor was achieved. The minor difference of MDL obtained from Fig. 7 and Fig. 8 resulted from the dilution process.

\section{Conclusions}

In conclusion, a sensitive QEPAS based $\mathrm{HCl}$ sensor using a QTF with $f_{0}$ of $30.72 \mathrm{kHz}$ was demonstrated. A pigtailed, near infrared, CW, TEC, DFB diode laser emitting at $1.74 \mu \mathrm{m}$ was employed as the laser excitation source. Wavelength modulation spectroscopy and a 2nd harmonic detection technique were used to reduce the sensor background noise and simplify data processing. A significant signal enhancement of 7.5 times was obtained when two mR were added to the QTF sensor architecture. For the $\mathrm{HCl}$ sensor system operating at atmospheric pressure, a 550 ppbv MDL at $5739.27 \mathrm{~cm}^{-1}$ was achieved when the modulation depth and data acquisition time were set to $0.23 \mathrm{~cm}^{-1}$ and $1 \mathrm{sec}$, respectively. Furthermore, the detection limit can be further improved when a QTF with a lower $f_{0}$ and multiple QTFs are adopted [20]. With a detection sensitivity at ppb concentration levels the reported $\mathrm{HCl}$ QEPAS based sensor is suitable for applications in environmental monitoring, atmospheric chemistry and industrial chemical analysis. 


\section{Acknowledgments}

This work was supported by the National Natural Science Foundation of China (Grant No. 61505041), the Natural Science Foundation of Heilongjiang Province of China (Grant No. F2015011), the General Financial Grant from the China Postdoctoral Science Foundation (Grant No. 2014M560262), the Special Financial Grant from the China Postdoctoral Science Foundation (Grant No. 2015T80350), the Special Financial Grant from the Heilongjiang Province Postdoctoral Foundation (Grant No. LBH-TZ0602), the Postdoctoral Fund of Heilongjiang Province (Grant No. LBH-Z14074), the Fundamental Research Funds for the Central Universities (Grant No. HIT. NSRIF. 2015044), and the National Key Scientific Instrument and Equipment Development Projects of China (Grant No. 2012YQ040164). Frank K. Tittel gratefully acknowledges the financial support from the US National Science Foundation ERC MIRTHE award and a grant C-0586 from the Welch Foundation.

\section{References}

[1] L.Wang, R.V. Kumar, Thick film miniaturized $\mathrm{HCl}$ gas sensor, Sens. Actuators B 98 (2004) 196-203.

[2] Z. S. Li, Z. W. Sun, B. Li, M. Alden, and M. Forsth, Spatially resolved trace detection of $\mathrm{HCl}$ in flames with mid-infrared polarization spectroscopy, Opt. Lett. 33(16) (2008) 1836-1838.

[3] Y. B. Huang, Y. A. Yang, G. X. He, S. Hashimoto, R. J. Gordon, State resolved translational energy distributions of $\mathrm{Cl}$ and $\mathrm{HCl}$ in the ultraviolet photodissociation of chloroethylenes, J. Chem. Phys. 103, (1995) 5476-5487.

[4] S. Kim, P. Klimecky, J. B. Jeffries, F. L. Terry, R. K. Hanson, In situ measurements of $\mathrm{HCl}$ during plasma etching of poly-silicon using a diode laser absorption sensor, Meas. Sci. Technol. 14, (2003) 1662-1670.

[5] H. Supriyatnoa, M. Yamashitaa, K. Nakagawab, Y. Sadaoka, Optochemical sensor for $\mathrm{HCl}$ gas based on tetraphenylporphyrin dispersed in styrene-acrylate copolymers: effects of glass transition temperature of matrix on $\mathrm{HCl}$ detection, Sens. Actuators B 85 (2002) 197-204.

[6] P. Ortwein, W. Woiwode, S. Fleck, M. Eberhard, T. Kolb, S. Wagner, M. Gisi, V. Ebert, Absolute diode laser-based in situ detection of $\mathrm{HCl}$ in gasification processes, Exp. Fluids 49 (2010) 961-968.

[7] J. Li, U. Parchatka, R. Königstedt, H. Fischer, Real-time measurements of atmospheric $\mathrm{CO}$ using a continuous-wave room temperature quantum cascade laser based spectrometer, Opt. Express 20 (2012) 7590-7601.

[8] Y. Y. Tang, W. Q. Liu, R. F. Kan, J. G. Liu, Y. B. He, Y. J. Zhang, Z.Y. Xu, J. Ruan, H. Geng, "Measurements of NO and CO in Shanghai urban atmosphere by using quantum cascade lasers," Opt. Express 19 (2011) 20224-20232.

[9] J. B. McManus, M. S. Zahniser, D. D. Nelson, Dual quantum cascade laser trace gas instrument with astigmatic Herriott cell at high pass number, Appl. Opt. 50 (2011) A74-A85.

[10] A. Elia, P. M. Lugarà, C. di Franco, V. Spagnolo, Photoacoustic techniques for 
trace gas sensing based on semiconductor laser sources. Sensors 9 (2009) 9616-9628. [11] A. A. Kosterev, Y. A. Bakhirkin, R. F. Curl, F. K. Tittel, Quartz-enhanced photoacoustic spectroscopy. Opt. Lett. 27 (2002) 1902-1904.

[12] A. A. Kosterev, F. K. Tittel, D. V. Serebryakov, A. L. Malinovsky, I. V. Morozov, Applications of quartz tuning forks in spectroscopic gas sensing, Review of Scientific Instruments, 76 (2005) 043105.

[13] S. Borri, P. Patimisco, A. Sampaolo, M. S. Vitiello, H. E. Beere, D. A. Ritchie, G. Scamarcio, V. Spagnolo, Terahertz quartz enhanced photo-acoustic sensor, Appl. Phys. Lett. 103 (2013) 021105.

[14] P. Patimisco, S. Borri, A. Sampaolo, H. E. Beere, D. A. Ritchie, M. S. Vitiello, G. Scamarcio, V. Spagnolo, Quartz enhanced photo-acoustic gas sensor based on custom tuning fork and terahertz quantum cascade laser, Analyst, 139 (2014) 2079-2087.

[15] H. P. Wu, A. Sampaolo, L. Dong, P. Patimisco, X. L. Liu, H. D. Zheng, X. K. Yin, W. G. Ma, L. Zhang, W. B. Yin, V. Spagnolo, S. T. Jia, F. K. Tittel, Quartz enhanced photoacoustic $\mathrm{H}_{2} \mathrm{~S}$ gas sensor based on a fiber-amplifier source and a custom tuning fork with large prong spacing, Appl. Phys. Lett. 107 (2015) 111104.

[16] W. Ren, L. Q. Luo, F. K. Tittel, Sensitive detection of formaldehyde using an interbandcascade laser near $3.6 \mu \mathrm{m}$, Sens. Actuators B 221 (2015) 1062-1068.

[17] Y. F. Ma, R. Lewicki, M. Razeghi, F. K. Tittel, QEPAS based ppb-level detection of $\mathrm{CO}$ and $\mathrm{N}_{2} \mathrm{O}$ using a high power CW DFB-QCL, Opt. Express, 21(1) (2013) 1008-1019.

[18] L. Dong, V. Spagnolo, R. Lewicki, F. K. Tittel, Ppb-level detection of nitric oxide using an external cavity quantum cascade laser based QEPAS sensor, Opt. Express, 19(24) (2011) 24037-24045.

[19] K. Liu, J. Li, L. Wang, T. Tan, W. Zhang, X. M. Gao, W. D. Chen, F. K. Tittel, Trace gas sensor based on quartz tuning fork enhanced laser photoacoustic spectroscopy, Appl. Phys. B 94 (2009) 527-533.

[20] Y.F. Ma, X. Yu, G. Yu, X.D. Li, J. B. Zhang, D. Y. Chen, R. Sun, F. K. Tittel, Multi-quartz-enhanced photoacoustic spectroscopy, Appl. Phys. Lett. 107 (2015) 021106.

\section{Biographies}

Yufei Ma received his Ph.D. degree in physical electronics from Harbin Institute of Technology, China, in 2013. From September 2010 to September 2011, he spent as a visiting scholar at Rice University, USA. Currently, he is a lecturer at the Harbin Institute of Technology, China. His research interests include optical sensors, trace gas detection, laser spectroscopy and optoelectronics.

Ying He received his B.S. degree in optoelectronic material and device from Harbin Engineering University, China, in 2015. He is now pursuing a M.S. degree of 
physical electronics from the Harbin Institute of Technology. His research interests include photoacoustic spectroscopy and optoelectronics techniques.

Xin Yu received her Ph.D. degree in physical electronics from Harbin Institute of Technology, China, in 2002. Now she is a professor at the Harbin Institute of Technology. Her research interests include laser detection, laser diagnostics, solid-state laser, laser ignition and optical sensors.

Rui Sun received his Ph.D. degree in thermal engineering from Harbin Institute of Technology in 1998. He is currently a professor in the College of Energy Science Engineering of the Harbin Institute of Technology. His research interests include clean coal combustion, alternative fuels (as biomass, waste etc.), optical diagnostics and laser spectroscopy.

Frank K. Tittel received his B.S. degree in 1955 and the Ph.D. degree in 1959 from Oxford University. He is the J.S. Abercrombie professor in the Department of Electrical Engineering at Rice University, Houston, TX USA. Professor Frank Tittel has been involved in many innovative developments in quantum electronics and laser technology since the discovery of the laser in 1960, with applications ranging from laser spectroscopy to environmental monitoring. The most recent designs utilize novel mid-infrared quantum cascade and interband lasers to achieve compact, robust instrumentation that can be deployed for field applications, such as by the US Environmental Protection Agency (EPA) for urban formaldehyde monitoring, methane and ethane detection funded by the US Department of Energy ARPA-E MONITOR program and by the US National Institute of Health for non-invasive NO and $\mathrm{CO}$ detection based on biomedical systems. 


\section{Biographies}

Yufei Ma received his Ph.D. degree in physical electronics from Harbin Institute of Technology, China, in 2013. From September 2010 to September 2011, he spent as a visiting scholar at Rice University, USA. Currently, he is a lecturer at the Harbin Institute of Technology, China. His research interests include optical sensors, trace gas detection, laser spectroscopy and optoelectronics.

Ying He received his B.S. degree in optoelectronic material and device from Harbin Engineering University, China, in 2015. He is now pursuing a M.S. degree of physical electronics from the Harbin Institute of Technology. His research interests include photoacoustic spectroscopy and optoelectronics techniques.

Xin Yu received her Ph.D. degree in physical electronics from Harbin Institute of Technology, China, in 2002. Now she is a professor at the Harbin Institute of Technology. Her research interests include laser detection, laser diagnostics, solid-state laser, laser ignition and optical sensors.

Rui Sun received his Ph.D. degree in thermal engineering from Harbin Institute of Technology in 1998. He is currently a professor in the College of Energy Science Engineering of the Harbin Institute of Technology. His research interests include clean coal combustion, alternative fuels (as biomass, waste etc.), optical diagnostics and laser spectroscopy.

Frank K. Tittel received his B.S. degree in 1955 and the Ph.D. degree in 1959 from Oxford University. He is the J.S. Abercrombie professor in the Department of Electrical Engineering at Rice University, Houston, TX USA. Professor Frank Tittel has been involved in many innovative developments in quantum electronics and laser technology since the discovery of the laser in 1960, with applications ranging from laser spectroscopy to environmental monitoring. The most recent designs utilize novel mid-infrared quantum cascade and interband lasers to achieve compact, robust instrumentation that can be deployed for field applications, such as by the US Environmental Protection Agency (EPA) for urban formaldehyde monitoring, methane and ethane detection funded by the US Department of Energy ARPA-E MONITOR program and by the US National Institute of Health for non-invasive NO and $\mathrm{CO}$ detection based on biomedical systems. 